\title{
Effects of Scatter Modeling on Time-Activity Curves Estimated Directly from Dynamic SPECT Projections
}

\author{
Bryan W. Reutter, Member, IEEE, Grant T. Gullberg, Fellow, IEEE, \\ and Ronald H. Huesman, Fellow, IEEE
}

\begin{abstract}
Quantitative analysis of uptake and washout of cardiac single photon emission computed tomography (SPECT) radiopharmaceuticals has the potential to provide better contrast between healthy and diseased tissue, compared to conventional reconstruction of static images. Previously, we used B-splines to model time-activity curves (TACs) for segmented volumes of interest and developed fast least-squares algorithms to estimate spline TAC coefficients and their statistical uncertainties directly from dynamic SPECT projection data. This previous work incorporated physical effects of attenuation and depth-dependent collimator response. In the present work, we incorporate scatter and use a computer simulation to study how scatter modeling affects directly estimated TACs and subsequent estimates of compartmental model parameters. An idealized single-slice emission phantom was used to simulate a $15 \mathrm{~min}$ dynamic ${ }^{99 \mathrm{~m}}$ Tc-teboroxime cardiac patient study in which 500,000 events containing scatter were detected from the slice. When scatter was modeled, unweighted leastsquares estimates of TACs had root mean square (RMS) error that was less than $0.6 \%$ for normal left ventricular myocardium, blood pool, liver, and background tissue volumes and averaged $3 \%$ for two small myocardial defects. When scatter was not modeled, RMS error increased to average values of $16 \%$ for the four larger volumes and $35 \%$ for the small defects. Noise-to-signal ratios (NSRs) for TACs ranged between 1-18\% for the larger volumes and averaged $110 \%$ for the small defects when scatter was modeled. When scatter was not modeled, NSR improved by average factors of $\mathbf{1 . 0 4}$ for the larger volumes and $\mathbf{1 . 2 5}$ for the small defects, as a result of the better-posed (though more biased) inverse problem. Weighted least-squares estimates of TACs had slightly better NSR and worse RMS error, compared to unweighted least-squares estimates. Compartmental model uptake and washout parameter estimates obtained from the TACs were less sensitive to whether or not scatter was modeled, compared to the TACs themselves.
\end{abstract}

\section{INTRODUCTION}

Q UANTITATIVE kinetic analysis of dynamic cardiac single photon emission computed tomography (SPECT) data has the potential to provide better contrast between healthy

This work was supported by the U.S. Department of Health and Human Services under grant R01-EB001970/R01-HL50663 and by the Director, Office of Science, Office of Biological and Environmental Research, Medical Sciences Division of the U.S. Department of Energy under contract DE-AC0376SF00098. This work was developed in part with the use of resources at the U.S. Department of Energy National Energy Research Scientific Computing (NERSC) Center.

The authors are with the Department of Nuclear Medicine and Functional Imaging, Lawrence Berkeley National Laboratory, University of California, Berkeley, CA 94720 USA (e-mail: bwreutter@lbl.gov). and diseased tissue, compared to static images. In particular, compartmental model parameters have physiological meaning and can provide a quantitative measure of tissue perfusion [1]. It has been hypothesized that compartmental analysis may also be useful for assessing tissue viability [2].

Previously, we used B-splines to model time courses of activity within segmented volumes of interest and developed fast least-squares algorithms to estimate spline model coefficients and their statistical uncertainties directly from dynamic SPECT projection data [3], [4]. We also studied how temporal modeling with splines affects the accuracy and precision of compartmental model parameters obtained from the timeactivity curve models [5]. This previous work incorporated physical effects of attenuation and depth-dependent collimator response. In the present work, we incorporate scatter and use a computer simulation to study how scatter modeling affects bias and variance of directly estimated time-activity curves and bias of compartmental model parameters obtained from the curves.

\section{Methods}

\section{A. Direct Temporal B-Spline Model Estimation}

Smooth time-activity curves for segmented volumes encompassing the projected field of view can be estimated directly from dynamic SPECT projection data as follows.

The time-activity curve for segmented volume $m$ is denoted by $A^{m}(t)$ and is modeled as a sum of temporal B-spline basis functions:

$$
A^{m}(t)=\sum_{n=1}^{N} a_{m n} V^{n}(t),
$$

where $a_{m n}$ are model coefficients, $V^{n}(t)$ are the B-spline basis functions [6], and $N$ is the number of basis functions. Splines with smaller support typically are used to model rapidly changing portions of curves, while splines with larger support are used to model slow changes (e.g., [7]).

The detected count rate at time $t$ along ray $i$ is modeled as

$$
P_{i}(t)=\sum_{m=1}^{M} U_{i}^{m}(t) A^{m}(t)=\sum_{m=1}^{M} \sum_{n=1}^{N} a_{m n} U_{i}^{m}(t) V^{n}(t),
$$

where $U_{i}^{m}(t)$ is the spatial projection, along ray $i$, of the indicator function for volume $m$ and $M$ is the number of 
segmented volumes encompassing the projected field of view. The factor $U_{i}^{m}(t)$ models physical effects such as attenuation, scatter, and depth-dependent collimator response.

The model for the dynamic projection data is obtained by integrating (2) over $L$ contiguous time intervals that span the data acquisition from time $t_{0}=0$ to time $t_{L}=T$ :

$$
p_{i l}=\sum_{m=1}^{M} \sum_{n=1}^{N} a_{m n} \int_{t_{l-1}}^{t_{l}} U_{i}^{m}(\tau) V^{n}(\tau) d \tau .
$$

If the time intervals are short enough so that each segmented volume projection function $U_{i}^{m}(t)$ is approximated well by a piecewise constant function with amplitude $u_{i l}^{m}$ during time interval $\left[t_{l-1}, t_{l}\right]$, then the model for the projection data can be simplified:

$$
p_{i l}=\sum_{m=1}^{M} \sum_{n=1}^{N} a_{m n} u_{i l}^{m} v_{l}^{n},
$$

where $v_{l}^{n}$ are the integrals $\int_{t_{l-1}}^{t_{l}} V^{n}(\tau) d \tau$ of the temporal Bspline basis functions.

The temporal spline model coefficients $a_{m n}$ are estimated by minimizing the weighted sum of squared differences between the measured and modeled projections:

$$
\chi^{2}=\sum_{i=1}^{I} \sum_{l=1}^{L} \frac{\left(p_{i l}^{*}-p_{i l}\right)^{2}}{W_{i l}}
$$

where $p_{i l}^{*}$ are the measured projections, $W_{i l}$ are weighting factors, and $I$ is the number of projection rays acquired simultaneously by the detector(s). Typically, the weighting factors $W_{i l}$ are estimated variances of the projections, or unity for unweighted fits. For a periodic (e.g., multi-rotation circular) orbit, the spline model coefficients $a_{m n}$, their covariance matrix, and the global precision of the time-activity curve models can be estimated with the use of fast methods that take advantage of periodicity in the $u_{i l}^{m}$ factors [3], [4].

\section{B. Nonlinear Compartmental Modeling}

Directly estimated spline models yield smooth time-activity curves that can have a variety of shapes. In many cases the relationship between the time-activity curves for the blood pool and a tissue volume of interest is described accurately by a compartmental model that has physiological meaning [1], [2].

For a one-compartment kinetic model (Fig. 1), the relationship between the blood input function, $B(t)$, and the activity in the tissue in volume $m, Q^{m}(t)$, is modeled to be

$$
\frac{d Q^{m}(t)}{d t}=k_{21}^{m} B(t)-k_{12}^{m} Q^{m}(t)
$$

where $k_{21}^{m}$ is the uptake rate parameter and $k_{12}^{m}$ is the washout rate parameter. For initial conditions of zero, the tissue activity is the convolution of the blood input function with a single decaying exponential:

$$
Q^{m}(t)=k_{21}^{m} \int_{0}^{t} B(\tau) e^{-k_{12}^{m}(t-\tau)} d \tau=k_{21}^{m} C^{m}(t)
$$

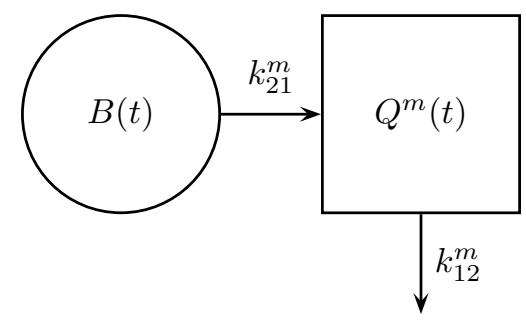

Fig. 1. Compartmental model for ${ }^{99 \mathrm{~m}} \mathrm{Tc}$-teboroxime in the myocardium.

Total activity in volume $m$ is given by $k_{21}^{m} C^{m}(t)+f_{v}^{m} B(t)$, where $f_{v}^{m}$ is the fraction of vasculature in the volume.

The program RFIT [8] is used to estimate compartmental model parameters $k_{21}^{m}, k_{12}^{m}$, and $f_{v}^{m}$ by minimizing the sum of squared differences between the spline and compartmental models:

$\chi_{m}^{2}=\sum_{l=1}^{L}\left\{\sum_{n=1}^{N} \hat{a}_{m n} v_{l}^{n}-\int_{t_{l-1}}^{t_{l}}\left[k_{21}^{m} \hat{C}^{m}(\tau)+f_{v}^{m} \hat{B}(\tau)\right] d \tau\right\}^{2}$,

where $\hat{a}_{m n}$ are values for spline model coefficients that minimize (5), $\sum_{n=1}^{N} \hat{a}_{m n} v_{l}^{n}$ is the integral of the temporal spline model for total activity in volume $m$ during time interval $\left[t_{l-1}, t_{l}\right], \hat{C}^{m}(\tau)$ is the convolution $\int_{0}^{\tau} \hat{B}\left(\tau^{\prime}\right) e^{-k_{12}^{m}\left(\tau-\tau^{\prime}\right)} d \tau^{\prime}$, and $\hat{B}(\tau)$ is derived from the temporal spline model for activity in the blood pool.

\section{Computer Simulation}

Simulated spatial distributions were obtained with the use of the idealized single-slice emission phantom shown in Fig. 2. The phantom contained $M=6$ circular volumes of interest: blood pool, three left ventricular tissue volumes (normal myocardium, septal defect, and lateral defect), liver, and background tissue. Each volume contained spatially uniform activity.

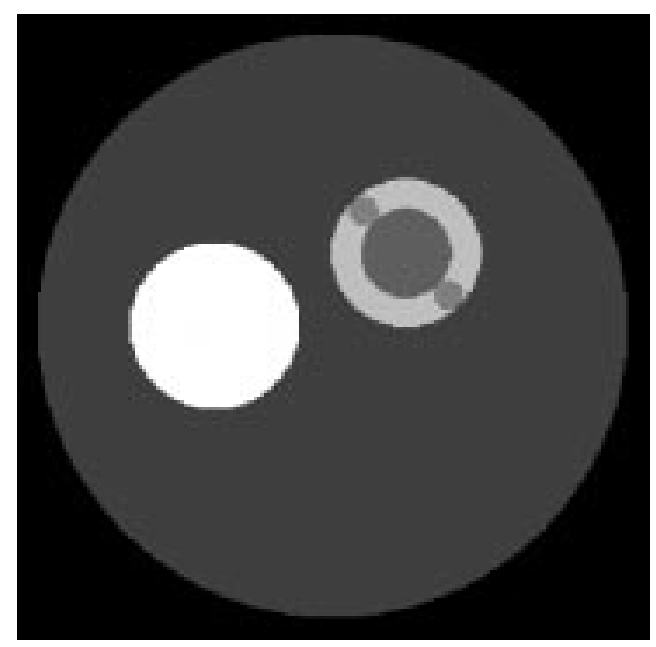

Fig. 2. Emission phantom. 


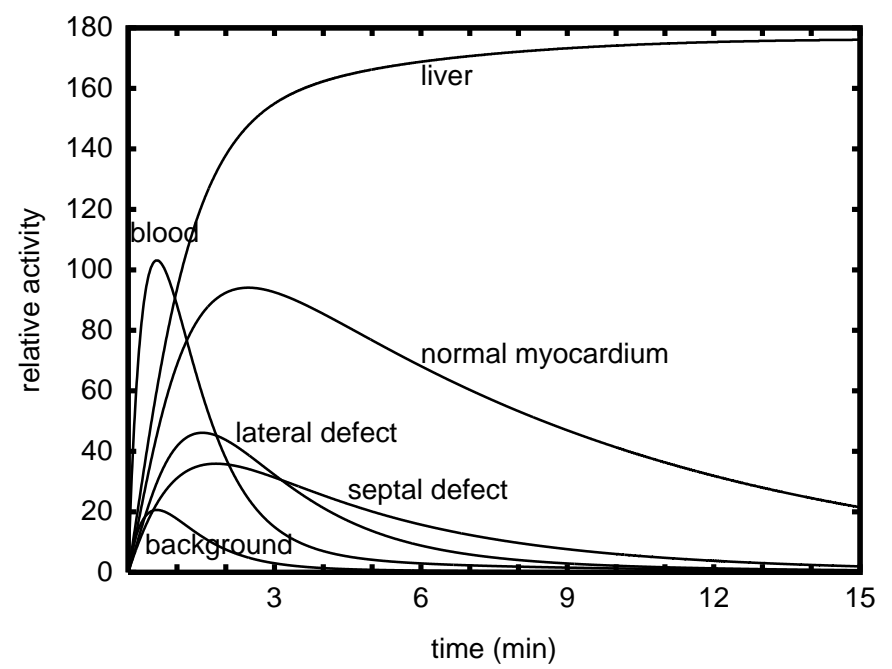

Fig. 3. Simulated ${ }^{99 m}$ Tc-teboroxime time-activity curves.

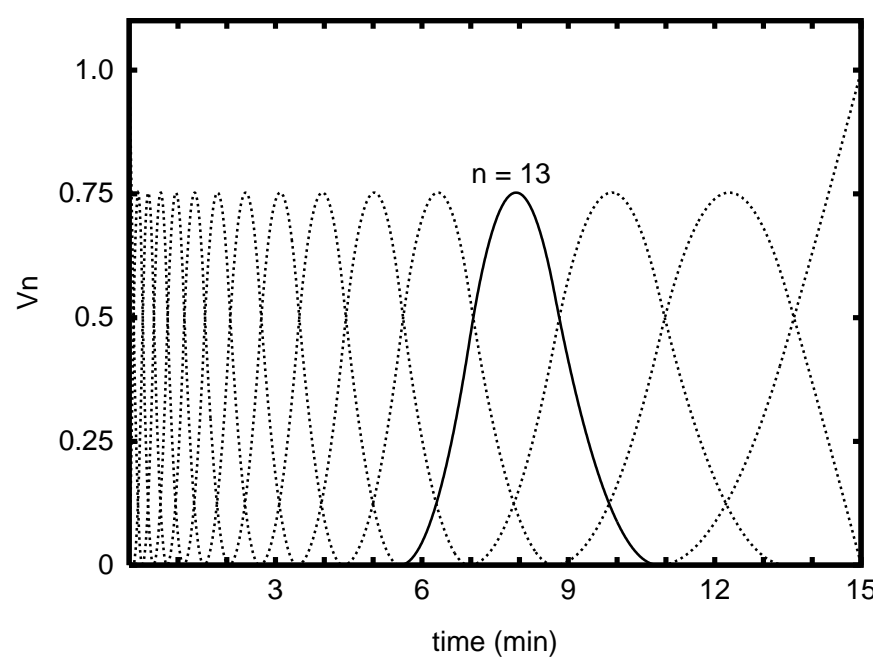

Fig. 4. Piecewise quadratic B-spline temporal basis functions used to model time-activity curves. The thirteenth spline is shown as a solid curve.

Simulated time-activity curves (Fig. 3) mimicked the kinetics of ${ }^{99 \mathrm{~m}}$ Tc-teboroxime [9]. The blood curve was used as the input function for one-compartment models that generated curves for the three myocardial tissue volumes and the liver. To make imaging the defects especially difficult, the defects were simulated to have both reduced uptake and accelerated washout, compared to the normal myocardium. The background tissue curve was proportional to the blood curve.

The simulated 15 min data acquisition with a single-detector system consisted of one $360^{\circ}$ rotation per minute, 120 projection angles per rotation, $I=50$ parallel projection rays per angle, and $L=1800$ time intervals. Projection bins were $6 \mathrm{~mm}$. Uniform attenuation and scatter at $140 \mathrm{keV}$ were simulated with the use of a ray-driven projector and analytic line integrals [10], [11]. Depth-dependent collimator response was not simulated. The blood input amplitude was adjusted so that 500,000 or $2,000,000$ events were detected. The scatter fraction was $15 \%$.
Time-activity curves were estimated directly from projection data with the use of the known spatial segmentation of the emission phantom and a set of $N=16$ temporal quadratic B-splines (Fig. 4). Curves were estimated both with and without incorporation of scatter effects in the $u_{i j}^{m}$ factors. Unweighted leastsquares (ULS) estimates were obtained, as well as weighted least-squares (WLS) estimates that used the known variances of the projections as weights $W_{i l}$ in (5).

\section{Results}

Fig. 5 shows ULS estimates of spline models for timeactivity curves obtained directly from projections. Proper scatter modeling yielded spline time-activity curves that closely fit the simulated curves. Failure to model scatter resulted in increased amplitudes for all curves, in order to account for the scattered events.

Table I shows root mean square (RMS) errors for timeactivity curves estimated from noiseless data, with and without scatter modeling. RMS error was calculated as the RMS difference between the estimated curve and the simulated curve, and was then normalized by the RMS value for the simulated curve and expressed as a percentage.

When ULS estimates were made and scatter was modeled, RMS error was less than $0.6 \%$ for the four larger volumes and ranged between $2-4 \%$ for the small myocardial defects. RMS error increased substantially when scatter was not modeled. Errors ranged between 14-20\% for the larger volumes and $25-45 \%$ for the small defects.

In most cases WLS estimates had larger RMS error than ULS estimates. When scatter was modeled, RMS error was still less than $1 \%$ for the larger volumes and ranged between $5-10 \%$ for the small defects. When scatter was not modeled, errors ranged between $12-20 \%$ for the larger volumes and $32-44 \%$ for the small defects.

Table I also shows noise-to-signal ratios (NSRs) that would be observed for time-activity curves estimated directly from noisy projections containing 500,000 or 2,000,000 events, with and without scatter modeling. NSR was calculated as the root mean value (across time) of expected squared differences between curves estimated from noisy and noiseless data, normalized by the RMS value for the noiseless curve and expressed as a percentage [4].

When ULS estimates were made based on 500,000 detected events and scatter was modeled, the liver and background tissue had the best NSR of about $1 \%$ because these volumes contributed the greatest numbers of events. NSR increased to $7 \%$ and $18 \%$ for the normal myocardium and blood pool, respectively. The myocardial defects had the worst NSR of about $110 \%$ because of their small size and low activity concentrations (Figs. 2, 3). When scatter was not modeled, NSR improved by average factors of 1.04 for the larger volumes and 1.25 for the small defects, as a result of the better-posed (though more biased) inverse problem.

NSR for WLS estimates improved by an average factor of 1.09, compared to ULS estimates. As expected for a linear 
TABLE I

EFFECTS OF SCATTER MODELING ON RMS ERRORS FOR CURVES ESTIMATED FROM NOISELESS DATA AND ON NOISE-TO-SIGNAL RATIOS FOR CURVES ESTIMATED FROM NOISY DATA CONTAINING 500,000 OR 2,000,000 DETECTED EVENTS. RESULTS ARE SHOWN FOR ULS AND WLS ESTIMATES.

\begin{tabular}{r|ccc|ccc}
\hline & \multicolumn{3}{|c|}{ scatter modeled } & \multicolumn{3}{c}{ scatter not modeled } \\
\cline { 2 - 7 } & $\begin{array}{c}\text { RMS error } \\
\text { noiseless } \\
(\%)\end{array}$ & $\begin{array}{c}\text { NSR } \\
0.5 \mathrm{M} \text { cts } \\
(\%)\end{array}$ & $\begin{array}{c}\text { NSR cts } \\
(\%)\end{array}$ & $\begin{array}{c}\text { RMS error } \\
\text { noiseless } \\
(\%)\end{array}$ & $\begin{array}{c}\text { NSR } \\
0.5 \mathrm{M} \text { cts } \\
(\%)\end{array}$ & $\begin{array}{c}\text { NSR cts } \\
(\%)\end{array}$ \\
\hline blood pool ULS & 0.57 & 17.6 & 8.8 & 19.4 & 16.2 & 8.1 \\
WLS & 0.94 & 16.6 & 8.3 & 19.5 & 15.2 & 7.6 \\
\hline normal myocardium ULS & 0.22 & 7.0 & 3.5 & 14.0 & 6.6 & 3.3 \\
WLS & 0.36 & 6.6 & 3.3 & 17.5 & 6.1 & 3.0 \\
\hline liver ULS & 0.03 & 0.9 & 0.4 & 14.8 & 0.9 & 0.4 \\
WLS & 0.05 & 0.8 & 0.4 & 16.6 & 0.8 & 0.4 \\
\hline Weptal defect ULS & 3.59 & 115 & 57.3 & 44.8 & 85.3 & 42.7 \\
WLS & 10.1 & 108 & 53.8 & 44.1 & 80.2 & 40.1 \\
\hline WLS & 2.24 & 109 & 54.7 & 24.6 & 94.0 & 47.0 \\
background ULS & 5.44 & 101 & 50.5 & 32.4 & 83.3 & 41.6 \\
\hline WLS & 0.46 & 1.3 & 0.7 & 14.7 & 1.3 & 0.6 \\
\hline
\end{tabular}
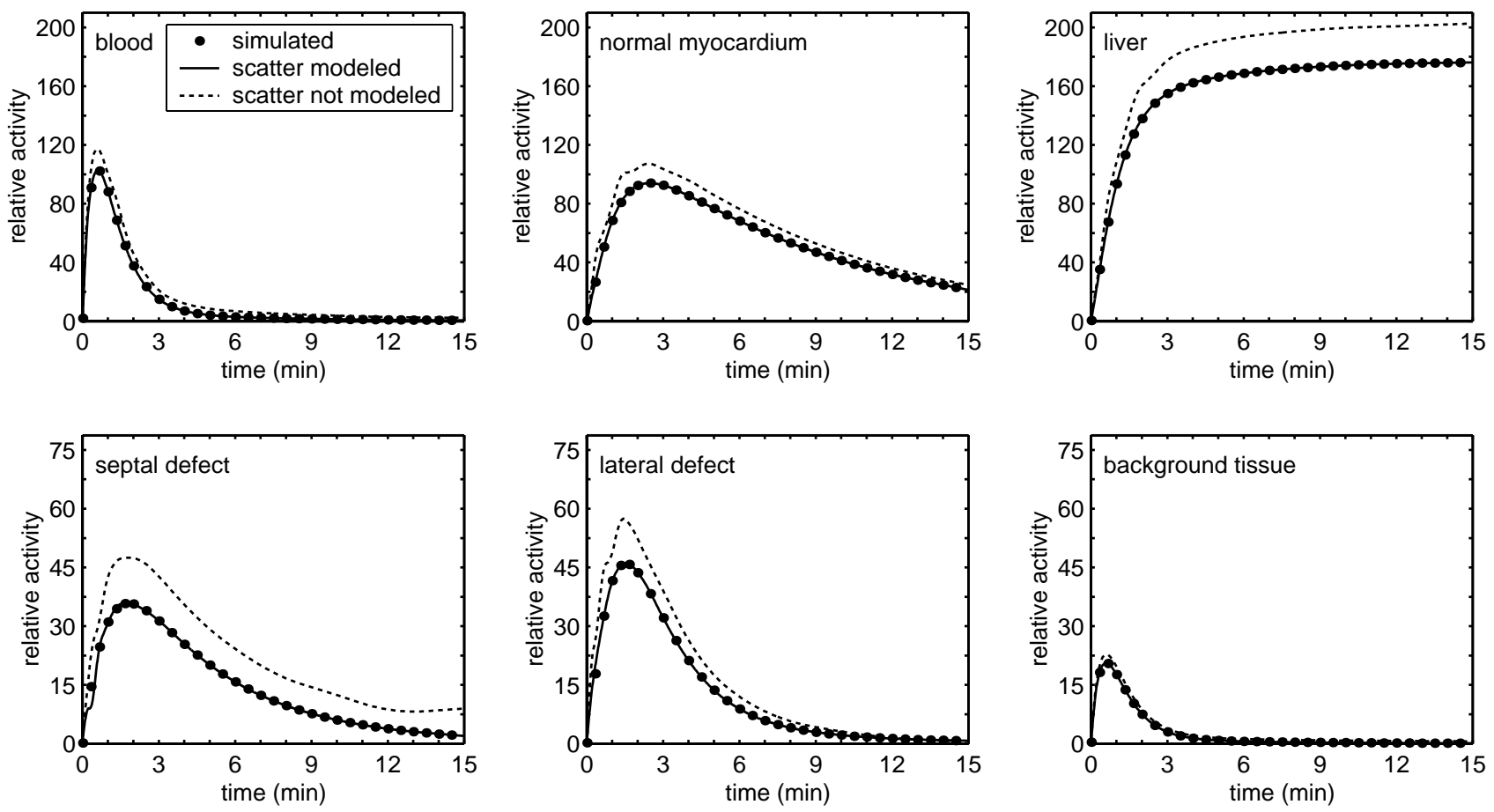

Fig. 5. ULS estimates of spline time-activity curves obtained from noiseless data with use of scatter modeling (solid line) and without use of scatter modeling (dashed line). Dots depict points on the simulated time-activity curves.

estimator, NSR improved by a factor of 2 when the number of detected events quadrupled.

Table II shows compartmental model parameters obtained from the spline time-activity curves. When ULS spline curve estimates were made and proper scatter modeling was used, uptake $\left(k_{21}\right)$ and washout $\left(k_{12}\right)$ parameter bias was no greater than $3 \%$. When scatter was not modeled, uptake parameter bias increased slightly to a maximum of $5 \%$. Washout parameter bias increased to about $10 \%$ for the defects and $20 \%$ for the normal myocardium. The apparent liver washout rate increased by nearly an order of magnitude but was still small. Thus, with the exception of the liver washout, the uptake and washout parameter bias was less sensitive to whether or not scatter was modeled, compared to the RMS error for the spline curves. Uptake and washout parameters were affected less by modeling errors because failure to model scatter caused only moderate changes in the relative amplitudes and shapes of the spline time-activity curves (Fig. 5).

When WLS spline curve estimates were made instead of ULS estimates, absolute changes in uptake and washout parameters 
TABLE II

EFFECTS OF SCATTER MODELING ON COMPARTMENTAL MODEL PARAMETERS OBTAINED FROM ULS AND WLS ESTIMATES OF TIME-ACTIVITY CURVES OBTAINED FROM NOISELESS DATA.

\begin{tabular}{r|ccc|ccc|ccc}
\hline & \multicolumn{3}{|c|}{ simulated value } & \multicolumn{3}{c|}{ scatter modeled } & \multicolumn{3}{c}{ scatter not modeled } \\
\cline { 2 - 11 } & \multicolumn{2}{|c|}{$k_{21} k_{12}$} & $f_{v}$ & \multicolumn{2}{c}{$k_{21}$} & $k_{12}$ & $f_{v}$ & \multicolumn{2}{c}{$k_{21}$} \\
$\left(\mathrm{~min}^{-1}\right)$ & & \multicolumn{2}{c|}{$\left.\left(\mathrm{min}_{12}\right)^{-1}\right)$} & $f_{v}$ \\
\hline normal myocardium ULS & 0.70 & 0.15 & 0.15 & 0.700 & 0.150 & 0.152 & 0.683 & 0.180 & 0.222 \\
WLS & 0.70 & 0.15 & 0.15 & 0.700 & 0.150 & 0.151 & 0.686 & 0.171 & 0.226 \\
\hline liver ULS & 0.90 & 0.002 & 0.20 & 0.901 & 0.0020 & 0.201 & 0.874 & 0.0131 & 0.253 \\
WLS & 0.90 & 0.002 & 0.20 & 0.901 & 0.0020 & 0.202 & 0.884 & 0.0123 & 0.249 \\
\hline WLS & 0.30 & 0.30 & 0.10 & 0.328 & 0.320 & 0.049 & 0.341 & 0.295 & 0.116 \\
\hline lateral defect ULS & 0.50 & 0.60 & 0.10 & 0.484 & 0.587 & 0.116 & 0.512 & 0.663 & 0.152 \\
WLS & 0.50 & 0.60 & 0.10 & 0.459 & 0.568 & 0.142 & 0.443 & 0.546 & 0.199 \\
\hline
\end{tabular}

for the normal myocardium and liver were small. Absolute changes were larger for the uptake and washout parameters for the defects, but bias was still less than $14 \%$ even when scatter was not modeled.

\section{Future Directions}

Work is underway to investigate the effects of scatter modeling in a simulated dynamic cone beam data acquisition that uses a more realistic 3-D spatial phantom. In particular, the effect of scatter modeling on the covariance of compartmental model parameters is being studied. Methods are also being developed for modeling scatter from relatively large volumes based on tracer kinetics.

\section{ACKNOWLEDGMENT}

This work was supported by the U.S. Department of Health and Human Services under grant R01-EB001970/R01HL50663 and by the Director, Office of Science, Office of Biological and Environmental Research, Medical Sciences Division of the U.S. Department of Energy under contract DE-AC03$76 \mathrm{SF} 00098$.

This work was developed in part with the use of resources at the U.S. Department of Energy National Energy Research Scientific Computing (NERSC) Center.

\section{REFERENCES}

[1] E. V. R. Di Bella, S. G. Ross, D. J. Kadrmas, H. S. Khare, P. E. Christian, S McJames, and G. T. Gullberg, "Compartmental modeling of technetium-99m-labeled teboroxime with dynamic single-photon emission computed tomography: Comparison with static thallium-201 in a canine model," Invest. Radiol., vol. 36, no. 3, pp. 178-185, 2001.

[2] G. T. Gullberg, R. H. Huesman, S. G. Ross, E. V. R. Di Bella, G. L. Zeng, B. W. Reutter, P. E. Christian, and S. A. Foresti, "Dynamic cardiac singlephoton emission computed tomography," in Nuclear Cardiology: State of the Art and Future Directions, B. L. Zaret and G. A. Beller, Eds., chapter 11, pp. 137-187. Mosby Inc., St. Louis, 1999.

[3] B. W. Reutter, G. T. Gullberg, and R. H. Huesman, "Direct leastsquares estimation of spatiotemporal distributions from dynamic SPECT projections using a spatial segmentation and temporal B-splines," IEEE Trans. Med. Imag., vol. 19, no. 5, pp. 434-450, 2000.

[4] B. W. Reutter, R. H. Huesman, and G. T. Gullberg, "Effects of temporal modelling on the statistical uncertainty of spatiotemporal distributions estimated directly from dynamic SPECT projections," Phys. Med. Biol., vol. 47, no. 15, pp. 2673-2683, 2002.
[5] B. W. Reutter, G. T. Gullberg, and R. H. Huesman, "Accuracy and precision of compartmental model parameters obtained from directly estimated dynamic SPECT time-activity curves," IEEE Trans. Nucl. Sci., in press.

[6] R. H. Bartels, J. C. Beatty, and B. A. Barsky, An Introduction to Splines for Use in Computer Graphics and Geometric Modeling, Morgan Kaufmann Publishers, Inc., Los Altos, CA, 1987.

[7] T. E. Nichols, J. Qi, E. Asma, and R. M. Leahy, "Spatiotemporal reconstruction of list-mode PET data," IEEE Trans. Med. Imag., vol. 21, no. 4, pp. 396-404, 2002.

[8] R. H. Huesman, B. L. Knittel, B. M. Mazoyer, P. G. Coxson, E. M. Salmeron, G. J. Klein, B. W. Reutter, and T. F. Budinger, "Notes on RFIT: A program for fitting compartmental models to region-of-interest dynamic emission tomography data," Tech. Rep. LBL-37621, Lawrence Berkeley National Laboratory, 1995.

[9] R. K. Narra, T. Feld, and A. D. Nunn, "Absorbed radiation dose to humans from technetium-99m-teboroxime," J. Nucl. Med., vol. 33, no. 1, pp. 88-93, 1992.

[10] R. H. Huesman, G. T. Gullberg, W. L. Greenberg, and T. F. Budinger, "RECLBL library users manual - Donner algorithms for reconstruction tomography," Publication PUB-214, Lawrence Berkeley Laboratory, 1977.

[11] A. Welch, G. T. Gullberg, P. E. Christian, F. L. Datz, and H. T. Morgan, "A transmission-map-based scatter correction technique for SPECT in inhomogeneous media," Med. Phys., vol. 22, no. 10, pp. 1627-1635, 1995.

\section{DISCLAIMER}

This document was prepared as an account of work sponsored by the United States Government. While this document is believed to contain correct information, neither the United States Government nor any agency thereof, nor The Regents of the University of California, nor any of their employees, makes any warranty, express or implied, or assumes any legal responsibility for the accuracy, completeness, or usefulness of any information, apparatus, product, or process disclosed, or represents that its use would not infringe privately owned rights. Reference herein to any specific commercial product, process, or service by its trade name, trademark, manufacturer, or otherwise, does not necessarily constitute or imply its endorsement, recommendation, or favoring by the United States Government or any agency thereof, or The Regents of the University of California. The views and opinions of authors expressed herein do not necessarily state or reflect those of the United States Government or any agency thereof, or The Regents of the University of California.

Ernest Orlando Lawrence Berkeley National Laboratory is an equal opportunity employer. 